\title{
FURTHER ACCELERATION OF SECANT-TYPE METHODS FOR SOLVING NONLINEAR EQUATIONS
}

\author{
R. THUKRAL \\ Padé Research Centre, 39 Deanswood Hill, Leeds, West Yorkshire, LS17 5JS, England \\ Corresponding author: rthukral@hotmail.co.uk
}

Received Apr. 17, 2020

ABSTRACT. In this paper, we present new Secant-type methods for finding simple root of nonlinear equations. It is proved that the new Secant-type methods have the convergence order of 2.83 or 2.55 requiring only two function evaluations per full iteration. Finally, the numerical examples are made with several other existing iterative methods to demonstrate the performance of the proposed methods. 2010 Mathematics Subject Classification. 65H05, 41A25.

Keywords: Newton method; Secant-type methods; Simple root; Nonlinear equations; Root-finding; Order of convergence.

\section{Introduction}

The development of numerical techniques for solving nonlinear equations is important research subject in numerical analysis $[4,5,12]$. In this paper, we consider iterative methods to find a simple root of a nonlinear equation $f(x)=0$ where $f: D \subset \Re \rightarrow \Re$ is a scalar function on an open interval $D$ and it is sufficiently smooth in a neighbourhood of the root. We present new three-point Secant-type iterative methods to find a simple root of the nonlinear equation. It is well established that the multipoint root-solvers is of great practical importance since it overcomes theoretical limits of one-point methods concerning the convergence order and computational efficiency. Recently, some

DOI: 10.28924/APJM/7-20 
modifications of the Secant-type methods for simple root have been proposed and analysed $[7-9,11]$. Hence, the purpose of this paper is to show further development of the Secant-type methods. The new Secant-type iterative methods are shown to have a better order of convergence then the methods considered in the previous studies [7-9]. In view of this fact, the proposed methods are significantly better when compared with the established methods [1-14].

We consider two well-known iterative methods for finding simple root of nonlinear equations are namely, the classical Secant method,

$x_{n+1}=x_{n}-\left[\frac{x_{n}-x_{n-1}}{f\left(x_{n}\right)-f\left(x_{n-1}\right)}\right] f\left(x_{n}\right)$,

and the classical Newton method

$$
x_{n+1}=x_{n}-\frac{f\left(x_{n}\right)}{f^{\prime}\left(x_{n}\right)},
$$

and their order of convergence is 1.62 and 2 respectively. However, for the purpose of this paper, we present new three-point secant-type methods for finding simple root of nonlinear equations.

The paper is organized as follows: Some essential definitions relevant to the present work are stated in the section 2. In section 3 we introduce four new three-point Secanttype methods and prove their order of convergence. In section 4, well-established Secant-type methods are stated, which will demonstrate the effectiveness of the new Secant-type iterative methods. Finally, in section 5, numerical comparisons are made to demonstrate the performance of the presented methods.

\section{Preliminaries}

In order to establish the order of convergence of an iterative method, following definitions are used $[4,5,12]$. 
Definition 1 Let $f(x)$ be a real-valued function with a root $\alpha$ and let $\left\{x_{n}\right\}$ be a sequence of real numbers that converge towards $\alpha$. The order of convergence $p$ is given by

$\lim _{n \rightarrow \infty} \frac{x_{n+1}-\alpha}{\left(x_{n}-\alpha\right)^{p}}=\zeta \neq 0$,

where $p \in \mathfrak{R}^{+}$and $\zeta$ is the asymptotic error constant.

Definition 2 Let $e_{k}=x_{k}-\alpha$ be the error in the $k$ th iteration, then the relation

$e_{k+1}=\zeta e_{k}^{p}+\mathrm{O}\left(e_{k}^{p+1}\right)$,

is the error equation. If the error equation exists, then $p$ is the order of convergence of the iterative method.

Definition 3 Let $r$ be the number of function evaluations of the method. The efficiency of the method is measured by the concept of efficiency index and defined as

$$
E I(r, p)=\sqrt[r]{p},
$$

where $p$ is the order of convergence of the method [4].

Definition 4 Suppose that $x_{n-1}, x_{n}$ and $x_{n+1}$ are three successive iterations closer to the root $\alpha$ of a nonlinear equation. Then the computational order of convergence [15] may be approximated by

$\mathrm{COC} \approx \frac{\ln \left|\left(x_{n+1}-\alpha\right)\left(x_{n}-\alpha\right)^{-1}\right|}{\ln \left|\left(x_{n}-\alpha\right)\left(x_{n-1}-\alpha\right)^{-1}\right|}$.

\section{Construction of the methods and convergence analysis}

In this section, we define the new three-point Secant-type iterative methods, methods having convergence order of 2.83 or 2.55 . To obtain the solution of (1), the new Secanttype methods requires two evaluations of functions and three particular starting points, ideally close to the simple root. The formulae of the new three-point Secant-type iterative methods for determining the simple root of (1) are; 
The first of the three-point Secant-type method is of convergence order 2.55 and is expressed as

$$
x_{n+1}=x_{n}-\frac{f\left(x_{n}\right)}{f^{\prime}\left(x_{n}\right)}-\left(\frac{1}{2}\right)\left(\frac{f\left(x_{n}\right)}{f^{\prime}\left(x_{n}\right)}\right)^{2}\left(\frac{\Delta_{7}}{f^{\prime}\left(x_{n}\right)}\right) .
$$

The next three of the three-point Secant-type methods are of convergence order 2.83 and they are expressed as

$$
\begin{aligned}
& x_{n+1}=x_{n}-\frac{f\left(x_{n}\right)}{f^{\prime}\left(x_{n}\right)}+\left[\frac{6 f\left(x_{n}\right) f^{\prime}\left(x_{n}\right)^{2}-3 f\left(x_{n}\right)^{2} \Delta_{8}}{6 f^{\prime}\left(x_{n}\right)^{3}-6 f\left(x_{n}\right) f^{\prime}\left(x_{n}\right) \Delta_{8}+f\left(x_{n}\right)^{2} \Delta_{10}}\right]+\frac{\Delta_{13}}{6}, \\
& x_{n+1}=x_{n}-\frac{f\left(x_{n}\right)}{f^{\prime}\left(x_{n}\right)}-\left(\frac{1}{2}\right)\left(\frac{f\left(x_{n}\right)}{f^{\prime}\left(x_{n}\right)}\right)^{2}\left(\frac{\Delta_{8}}{f^{\prime}\left(x_{n}\right)}\right)+\left(\frac{\Delta_{13}}{6}\right), \\
& x_{n+1}=x_{n}-\frac{f\left(x_{n}\right)}{f^{\prime}\left(x_{n}\right)}+\left[\frac{6 f\left(x_{n}\right) f^{\prime}\left(x_{n}\right)-3 f\left(x_{n}\right)^{2} \Delta_{8}}{6 f^{\prime}\left(x_{n}\right)^{3}-6 f\left(x_{n}\right) f^{\prime}\left(x_{n}\right) \Delta_{8}+f\left(x_{n}\right)^{2} \Delta_{10}+\Delta_{14}}\right],
\end{aligned}
$$

where

$$
\begin{aligned}
& \Delta_{1}=\frac{f\left(x_{n}\right)-f\left(x_{n-1}\right)}{x_{n}-x_{n-1}}, \\
& \Delta_{2}=\frac{f\left(x_{n-1}\right)-f\left(x_{n-2}\right)}{x_{n-1}-x_{n-2}}, \\
& \Delta_{3}=\frac{f\left(x_{n}\right)-f\left(x_{n-2}\right)}{x_{n}-x_{n-2}}, \\
& \Delta_{4}=\frac{f^{\prime}\left(x_{n}\right)-f^{\prime}\left(x_{n-1}\right)}{x_{n}-x_{n-1}}, \\
& \Delta_{5}=\frac{f^{\prime}\left(x_{n-1}\right)-f^{\prime}\left(x_{n-2}\right)}{x_{n-1}-x_{n-2}}, \\
& \Delta_{6}=\frac{f^{\prime}\left(x_{n}\right)-f^{\prime}\left(x_{n-2}\right)}{x_{n}-x_{n-2}}, \\
& \Delta_{7}=\Delta_{4}-\Delta_{5}+\Delta_{6},
\end{aligned}
$$




$$
\begin{aligned}
& \Delta_{8}=\frac{4 f^{\prime}\left(x_{n}\right)+2 f^{\prime}\left(x_{n-1}\right)-6 \Delta_{1}}{x_{n}-x_{n-1}}, \\
& \Delta_{9}=\frac{4 f^{\prime}\left(x_{n}\right)+2 f^{\prime}\left(x_{n-2}\right)-6 \Delta_{3}}{x_{n}-x_{n-2}},
\end{aligned}
$$

$$
\Delta_{10}=\frac{\Delta_{4}-\Delta_{8}}{x_{n}-x_{n-1}},
$$

$$
\Delta_{11}=\frac{\Delta_{6}-\Delta_{9}}{x_{n-2}-x_{n}},
$$

$\Delta_{12}=\frac{\Delta_{11}+\Delta_{10}}{x_{n-1}-x_{n-2}}$,

$\Delta_{13}=\left(\frac{\Delta_{12}}{f^{\prime}\left(x_{n}\right)}\right)\left(\frac{f\left(x_{n-1}\right)}{f^{\prime}\left(x_{n-1}\right)}\right)^{2}\left(\frac{f\left(x_{n}\right)}{f^{\prime}\left(x_{n}\right)}\right)^{2}$,

$\Delta_{14}=\left(\Delta_{12} f\left(x_{n}\right) f^{\prime}\left(x_{n}\right)\right)\left(\frac{f\left(x_{n-1}\right)}{f^{\prime}\left(x_{n-1}\right)}\right)^{2}$.

$x_{-1}, x_{0}, x_{1}$ are the initial points and provided that the denominators of (7)-(24) are not equal to zero. It is important to verify our finding and prove the order of convergence of the new three-point Secant-type iterative methods.

\section{Theorem 1}

Let $f: D \subset \Re \rightarrow \Re$ be a sufficiently differentiable function and let for an open interval $D$ has $\alpha \in D$ be a simple zero of $f(x)=0$ in an open interval $D$, with $f^{\prime}(x) \neq 0$ in $D$. If the initial points $x_{-1}, x_{0}$ and $x_{1}$ are sufficiently close to $\alpha$, then the asymptotic convergence order of the new methods defined by (8)-(10) is 2.83 .

\section{Proof}

Let $\alpha$ be a simple root of $f(x)$, i.e. $f(\alpha)=0$ and $f^{\prime}(\alpha) \neq 0$, and the errors at $(k-1), k$ and $(k+1)$ iteration are expressed as $e_{n-2}=x_{n-2}-\alpha, e_{n-1}=x_{n-1}-\alpha, e_{n}=x_{n}-\alpha$ and $e_{n+1}=x_{n+1}-\alpha$, respectively. 
Using Taylor expansion and taking into account that $f(\alpha)=0$, we have

$$
\begin{aligned}
& f\left(x_{n}\right)=c_{1} e_{n}+c_{2} e_{n}^{2}+c_{3} e_{n}^{3}+c_{4} e_{n}^{4}+\cdots \\
& f\left(x_{n-1}\right)=c_{1} e_{n-1}+c_{2} e_{n-1}^{2}+c_{3} e_{n-1}^{3}+c_{4} e_{n-1}^{4}+\cdots \\
& f\left(x_{n-2}\right)=c_{1} e_{n-2}+c_{2} e_{n-2}^{2}+c_{3} e_{n-2}^{3}+c_{4} e_{n-2}^{4}+\cdots \\
& f^{\prime}\left(x_{n}\right)=c_{1}+2 c_{2} e_{n}+3 c_{3} e_{n}^{2}+4 c_{4} e_{n}^{3}+\cdots \\
& f^{\prime}\left(x_{n-1}\right)=c_{1}+2 c_{2} e_{n-1}+3 c_{3} e_{n-1}^{2}+4 c_{4} e_{n-1}^{3}+\cdots \\
& f^{\prime}\left(x_{n-2}\right)=c_{1}+2 c_{2} e_{n-2}+3 c_{3} e_{n-2}^{2}+4 c_{4} e_{n-2}^{3}+\cdots
\end{aligned}
$$

where

$$
c_{k}=\frac{f^{(k)}(\alpha)}{(k !)}, \quad \text { for } k=1,2,3,4, \ldots .
$$

Using (25)-(30), we obtain

$$
\begin{aligned}
& \Delta_{1}=c_{1}+\left(e_{n}+e_{n-1}\right) c_{2}+\left(e_{n}^{2}+e_{n-1} e_{n}+e_{n-1}^{2}\right) c_{3}+\cdots \\
& \Delta_{2}=c_{1}+\left(e_{n-1}+e_{n-2}\right) c_{2}+\left(e_{n-1}^{2}+e_{n-1} e_{n-2}+e_{n-2}^{2}\right) c_{3}+\cdots \\
& \Delta_{3}=c_{1}+\left(e_{n}+e_{n-2}\right) c_{2}+\left(e_{n}^{2}+e_{n} e_{n-2}+e_{n-2}^{2}\right) c_{3}+\cdots \\
& \Delta_{4}=2 c_{2}+3\left(e_{n}+e_{n-1}\right) c_{3}+4\left(e_{n}^{2}+e_{n} e_{n-1}+e_{n-1}^{2}\right) c_{4}+\cdots \\
& \Delta_{5}=2 c_{2}+3\left(e_{n-1}+e_{n-2}\right) c_{3}+4\left(e_{n-1}^{2}+e_{n-1} e_{n-2}+e_{n-2}^{2}\right) c_{4}+\cdots \\
& \Delta_{6}=2 c_{2}+3\left(e_{n}+e_{n-2}\right) c_{3}+4\left(e_{n}^{2}+e_{n} e_{n-2}+e_{n-2}^{2}\right) c_{4}+\cdots \\
& \Delta_{7}=2 c_{2}+3 c_{3} e_{n}+4\left(8 e_{n}^{2}+4 e_{n} e_{n-1}+4 e_{n} e_{n-2}-4 e_{n-1} e_{n-2}\right) c_{4}+\cdots \\
& \Delta_{8}=2 c_{2}+\left(6 c_{3}+4 e_{n-1} c_{4}+12 e_{n-1}^{2} c_{5}\right) e_{n}+\cdots \\
& \Delta_{9}=2 c_{2}+\left(6 c_{3}+4 e_{n-2} c_{4}+12 e_{n-2}^{2} c_{5}\right) e_{n}+\cdots \\
& \Delta_{10}=-3 c_{3}-6\left(e_{n-1}+e_{n}\right) c_{4}+\cdots \\
& \Delta_{11}=3 c_{3}+6\left(e_{n-2}+e_{n}\right) c_{4}+\cdots \\
& \Delta_{12}=-3 c_{4}+6\left(e_{n-2}+e_{n-1}+3 e_{n}\right) c_{5}+\cdots
\end{aligned}
$$




$$
\begin{aligned}
& \Delta_{13}=-6 \frac{c_{4}}{c_{1}} e_{n-1}^{2} e_{n}^{2}+6 \frac{c_{5}}{c_{1}} e_{n-2} e_{n-1}^{2} e_{n}^{2}+\cdots \\
& \Delta_{14}=-6 c_{4} c_{1}^{2} e_{n-1}^{2} e_{n}+6 c_{5} c_{1}^{2} e_{n-2} e_{n-1}^{2} e_{n}+\cdots
\end{aligned}
$$

Substituting the appropriate expressions in (8),

$$
e_{n+1}=e_{n}-\frac{f\left(x_{n}\right)}{f^{\prime}\left(x_{n}\right)}+\left[\frac{6 f\left(x_{n}\right) f^{\prime}\left(x_{n}\right)^{2}-3 f\left(x_{n}\right)^{2} \Delta_{8}}{6 f^{\prime}\left(x_{n}\right)^{3}-6 f\left(x_{n}\right) f^{\prime}\left(x_{n}\right) \Delta_{8}+f\left(x_{n}\right)^{2} \Delta_{10}}\right]+\frac{\Delta_{13}}{6},
$$

Simplifying, we obtain the error equation for the new three-point Secant-type iterative method, given by (46) is

$$
e_{n+1}=\left(\frac{c_{5}}{c_{1}}\right) e_{n}^{2} e_{n-1}^{2} e_{n-2}+\cdots
$$

In order to prove the order of convergence of (47) and we defining positive real terms of

$$
\begin{aligned}
& R_{n}, \mathrm{R}_{n-1} \text { and } \mathrm{R}_{n-2} \text { as } \\
& R_{n}=\frac{\left|e_{n+1}\right|}{\left|e_{n}^{m}\right|}, \quad R_{n-1}=\frac{\left|e_{n}\right|}{\left|e_{n-1}^{m}\right|} \quad R_{n-2}=\frac{\left|e_{n-1}\right|}{\left|e_{n-2}^{m}\right|},
\end{aligned}
$$

The error terms of $R_{n-2}$ are given as

$$
\begin{aligned}
& \left|e_{n-1}\right|=R_{n-2}\left|e_{n-2}^{m}\right| \\
& \left|e_{n}\right|=R_{n-1}\left|e_{n-1}^{m}\right|=R_{n-1} R_{n-2}^{m}\left|e_{n-2}^{m^{2}}\right| \\
& \left|e_{n+1}\right|=R_{n}\left|e_{n}^{m}\right|=\left(R_{n} R_{n-1}^{m} R_{n-2}^{m^{2}}\right)\left|e_{n-2}^{m^{3}}\right| .
\end{aligned}
$$

It is obtained from (47) that

$$
\begin{aligned}
& \frac{\left|e_{n+1}\right|}{\left|e_{n}^{2}\right|\left|e_{n-1}^{2}\right|\left|e_{n-2}\right|}=\left|\left(\frac{c_{5}}{c_{1}}\right)\right|, \\
& \frac{\left|e_{n+1}\right|}{\left|e_{n}^{2}\right|\left|e_{n-1}^{2}\right|\left|e_{n-2}\right|}=\left(R_{n} R_{n-1}^{m-2} R_{n-2}^{m^{2}-2 m-2}\right)\left|e_{n-2}^{m^{3}-2 m^{2}-2 m-1}\right|=\left|\left(\frac{c_{5}}{c_{1}}\right)\right| .
\end{aligned}
$$

In order to satisfy the asymptotic equation (53), the power of the error term shall approach zero, that is $m^{3}-2 m^{2}-2 m-1=0$. 
The roots of the cubic equation (54) are;

$m=2.831177, \quad m=-0.415588-0.424848 i \quad m=-0.415588+0.424848 i$

The order of convergence of the new three-point Secant-type method is determined by the positive root of (54). Hence, the new three-point Secant-type method defined by (8) has a convergence order of 2.83. This completes the proof.

We repeat the procedure to prove the error equation of the Secant-type methods given by (9) and (10). Consequently, the error equations of the new three-point Secant-type method defined by (9) and (10) is identical to the error equation of (8). Hence, the new three-point Secant-type method defined by (9) and (10) have a convergence order of 2.83 .

\section{Theorem 2}

Let $f: D \subset \mathfrak{R} \rightarrow \mathfrak{R}$ be a sufficiently differentiable function and let for an open interval $D$ has $\alpha \in D$ be a simple zero of $f(x)=0$ in an open interval $D$, with $f^{\prime}(x) \neq 0$ in $D$. If the initial points $x_{0}$ and $x_{1}$ are sufficiently close to $\alpha$, then the asymptotic convergence order of the new Secant-type iterative method defined by (7) is 2.55 .

\section{Proof}

Using the similar procedure of the previous proof, we substitute the appropriate expressions in (16) and obtain

$$
e_{n+1}=e_{n}-\frac{f\left(x_{n}\right)}{f^{\prime}\left(x_{n}\right)}-\left(\frac{1}{2}\right)\left(\frac{f\left(x_{n}\right)}{f^{\prime}\left(x_{n}\right)}\right)^{2}\left(\frac{\Delta_{7}}{f^{\prime}\left(x_{n}\right)}\right),
$$

Simplifying, we obtain the error equation for the new three-point Secant-type iterative method is

$$
e_{n+1}=\left(\frac{2 c_{4}}{c_{1}}\right) e_{n}^{2} e_{n-1} e_{n-2}+\cdots .
$$

It is obtained from (57) that 


$$
\begin{aligned}
& \frac{\left|e_{n+1}\right|}{\left|e_{n}^{2}\right|\left|e_{n-1}\right|\left|e_{n-2}\right|}=\left|\left(\frac{2 c_{4}}{c_{1}}\right)\right|, \\
& \frac{\left|e_{n+1}\right|}{\left|e_{n}^{2}\right|\left|e_{n-1}\right|\left|e_{n-2}\right|}=\left(R_{n} R_{n-1}^{m-2} R_{n-2}^{m^{2}-2 m-1}\right)\left|e_{n-2}^{m^{3}-2 m^{2}-m-1}\right|=\mid\left(\frac{2 c_{4}}{c_{1}}\right) .
\end{aligned}
$$

In order to satisfy the asymptotic equation (59), the power of the error term shall approach zero, that is

$m^{3}-2 m^{2}-m-1=0$.

The roots of the cubic equation (60) are;

$m=2.546818, \quad m=-0.273409-0.563821 i \quad m=-0.273409+0.563821 i$

The order of convergence of the new three-point Secant-type methods is determined by the positive root of (60). Hence, the new three-point Secant-type methods defined by (7) has a convergence order of 2.55 .

\section{The established methods}

For the purpose of comparison, three particular well-established iterative methods [12] are considered and they are given below,

$$
\begin{aligned}
& x_{n+1}=x_{n}-\frac{f\left(x_{n}\right)}{f^{\prime}\left(x_{n}\right)}-\left(\frac{1}{2}\right)\left(\frac{f\left(x_{n}\right)}{f^{\prime}\left(x_{n}\right)}\right)^{2}\left(\frac{\Delta_{4}}{f^{\prime}\left(x_{n}\right)}\right), \\
& x_{n+1}=x_{n}-\frac{f\left(x_{n}\right)}{f^{\prime}\left(x_{n}\right)}+\left[\frac{f\left(x_{n}\right)^{2}}{f\left(x_{n}\right)-f\left(x_{n-1}\right)}\right]\left(\frac{2}{f^{\prime}\left(x_{n}\right)}+\frac{1}{f^{\prime}\left(x_{n-1}\right)}-\frac{3}{\Delta_{1}}\right), \\
& x_{n+1}=x_{n}-\frac{f\left(x_{n}\right)}{f^{\prime}\left(x_{n}\right)}-\left(\frac{1}{2}\right)\left(\frac{f\left(x_{n}\right)}{f^{\prime}\left(x_{n}\right)}\right)^{2}\left(\frac{\Delta_{8}}{f^{\prime}\left(x_{n}\right)}\right) .
\end{aligned}
$$

\section{Remark}

The new three-point Secant-type methods requires 2 function evaluations and have the order of convergence 2.83 or 2.55 . To determine the efficiency index of the new threepoint Secant-type methods, definition 3 will be used. Hence, the efficiency index of the new Secant-type iterative methods given by $(8)-(10)$ is $E I(2.83,2) \approx 1.683$, the efficiency 
index of the new 2.55-order Secant-type iterative method given by (7) is $E I(2.55,2) \approx 1.596$, the efficiency index of the iterative method given by (62) is $E I(2.41,2) \approx 1.55$, the efficiency index of the iterative methods given by (63) and (64) is $E I(2.732,2) \approx 1.653$, the efficiency index of the classical Secant method given by (1) is $\operatorname{EI}(1.618,1) \approx 1.618$, and finally the efficiency index of the Newton method given by (2) is $E I(2,2) \approx 1.414$.

\section{Numerical experiments}

In this section we check the effectiveness of the new Secant-type iterative methods introduced in this paper and compare them with the established iterative methods. The difference between the simple root $\alpha$ and the approximation $x_{n}$ for test function with starting points $x_{-1}, x_{0}, x_{1}$ is displayed in tables. Furthermore, the computational order of convergence approximations is displayed in tables and we observe that this perfectly coincides with the theoretical result. The numerical computations listed in the table was performed on an algebraic system called Maple and the errors displayed are of absolute value. We test the different iterative methods using the following smooth functions.

\section{Numerical example 1}

We demonstrate the order of convergence of the new three-point Secant-type iterative methods for the following nonlinear equation

$$
f(x)=\exp \left(x^{2}+7 x-30\right)-1,
$$

having the exact value of the simple root of (65) is $\alpha=3$ In Table 1 the errors obtained by the methods described are based on the starting points $x_{-1}=2.85, x_{0}=2.9, \quad x_{1}=2.95$. 
Table 1 Errors occurring in the approximation of the simple root of nonlinear equation (65)

\begin{tabular}{ccccccc}
\hline methods & $\left|x_{2}-\alpha\right|$ & $\left|x_{3}-\alpha\right|$ & $\left|x_{4}-\alpha\right|$ & $\left|x_{5}-\alpha\right|$ & $f\left(x_{5}\right)$ & COC \\
$(1)$ & $0.461 \mathrm{e}-1$ & $0.148 \mathrm{e}-1$ & $0.414 \mathrm{e}-2$ & $0.420 \mathrm{e}-3$ & $0.547 \mathrm{e}-2$ & 1.1978 \\
$(2)$ & $0.206 \mathrm{e}-1$ & $0.256 \mathrm{e}-2$ & $0.426 \mathrm{e}-4$ & $0.120 \mathrm{e}-7$ & $0.155 \mathrm{e}-6$ & 2.0000 \\
$(62)$ & $0.323 \mathrm{e}-2$ & $0.163 \mathrm{e}-4$ & $0.372 \mathrm{e}-10$ & $0.984 \mathrm{e}-24$ & $0.128 \mathrm{e}-22$ & 2.3913 \\
$(7)$ & $0.896 \mathrm{e}-2$ & $0.174 \mathrm{e}-5$ & $0.220 \mathrm{e}-12$ & $0.143 \mathrm{e}-30$ & $0.186 \mathrm{e}-29$ & 2.5827 \\
$(63)$ & $0.191 \mathrm{e}-2$ & $0.665 \mathrm{e}-5$ & $0.110 \mathrm{e}-12$ & $0.301 \mathrm{e}-33$ & $0.391 \mathrm{e}-32$ & 2.7569 \\
$(64)$ & $0.110 \mathrm{e}-1$ & $0.684 \mathrm{e}-4$ & $0.335 \mathrm{e}-10$ & $0.516 \mathrm{e}-27$ & $0.670 \mathrm{e}-26$ & 2.7507 \\
$(8)$ & $0.591 \mathrm{e}-2$ & $0.239 \mathrm{e}-5$ & $0.138 \mathrm{e}-14$ & $0.252 \mathrm{e}-40$ & $0.328 \mathrm{e}-39$ & 2.8493 \\
$(9)$ & $0.224 \mathrm{e}-1$ & $0.974 \mathrm{e}-3$ & $0.631 \mathrm{e}-7$ & $0.122 \mathrm{e}-19$ & $0.159 \mathrm{e}-18$ & 2.7001 \\
$(10)$ & $0.995 \mathrm{e}-3$ & $0.113 \mathrm{e}-6$ & $0.300 \mathrm{e}-18$ & $0.462 \mathrm{e}-51$ & $0.601 \mathrm{e}-50$ & 2.8228 \\
\hline
\end{tabular}

\section{Numerical example 2}

We demonstrate the order of convergence of the new three-point Secant-type iterative methods for the following nonlinear equation

$$
f(x)=(x-2)\left(x^{10}+x+1\right) \exp (-x-1),
$$

having exact value of the simple root of (66) is $\alpha=2$. In Table 2 the errors obtained by the methods described are based on the starting points $x_{-1}=2.003, \quad x_{0}=2.002, \quad x_{1}=2.001 .$.

Table 2 Errors occurring in the approximation of the simple root of nonlinear equation (66)

\begin{tabular}{ccccccc}
\hline methods & $\left|x_{2}-\alpha\right|$ & $\left|x_{3}-\alpha\right|$ & $\left|x_{4}-\alpha\right|$ & $\left|x_{5}-\alpha\right|$ & $f\left(x_{5}\right)$ & COC \\
$(1)$ & $0.792 \mathrm{e}-5$ & $0.315 \mathrm{e}-7$ & $0.994 \mathrm{e}-12$ & $0.125 \mathrm{e}-18$ & $0.638 \mathrm{e}-17$ & 1.6522 \\
$(2)$ & $0.397 \mathrm{e}-5$ & $0.628 \mathrm{e}-10$ & $0.157 \mathrm{e}-19$ & $0.983 \mathrm{e}-39$ & $0.503 \mathrm{e}-37$ & 2.0000 \\
$(62)$ & $0.148 \mathrm{e}-7$ & $0.221 \mathrm{e}-17$ & $0.733 \mathrm{e}-42$ & $0.120 \mathrm{e}-100$ & $0.614 \mathrm{e}-99$ & 2.4151 \\
$(7)$ & $0.248 \mathrm{e}-7$ & $0.153 \mathrm{e}-19$ & $0.709 \mathrm{e}-49$ & $0.232 \mathrm{e}-124$ & $0.119 \mathrm{e}-122$ & 2.5504 \\
$(63)$ & $0.250 \mathrm{e}-7$ & $0.117 \mathrm{e}-18$ & $0.161 \mathrm{e}-50$ & $0.670 \mathrm{e}-137$ & $0.342 \mathrm{e}-135$ & 2.7377 \\
$(64)$ & $0.248 \mathrm{e}-7$ & $0.411 \mathrm{e}-20$ & $0.631 \mathrm{e}-55$ & $0.409 \mathrm{e}-150$ & $0.209 \mathrm{e}-148$ & 2.7315 \\
$(8)$ & $0.101 \mathrm{e}-7$ & $0.144 \mathrm{e}-22$ & $0.932 \mathrm{e}-64$ & $0.805 \mathrm{e}-181$ & $0.412 \mathrm{e}-179$ & 2.8303 \\
$(9)$ & $0.248 \mathrm{e}-7$ & $0.406 \mathrm{e}-21$ & $0.445 \mathrm{e}-60$ & $0.357 \mathrm{e}-170$ & $0.182 \mathrm{e}-168$ & 2.8330 \\
$(10)$ & $0.101 \mathrm{e}-7$ & $0.122 \mathrm{e}-22$ & $0.932 \mathrm{e}-64$ & $0.805 \mathrm{e}-181$ & $0.412 \mathrm{e}-179$ & 2.8303 \\
\hline
\end{tabular}




\section{Numerical example 3}

We demonstrate the order of convergence of the new three-point Secant-type iterative methods for the following nonlinear equation

$$
f(x)=\left[\exp (x) \sin (x)+\ln \left(1+x^{2}\right)\right],
$$

having exact value of the simple root of (67) is $\alpha=0$. In Table 3 the errors obtained by the methods described are based on the starting points $x_{-1}=\frac{1}{10}, x_{0}=\frac{1}{11}, x_{1}=\frac{1}{12}$.

Table 3 Errors occurring in the approximation of the simple root of nonlinear equation (67)

\begin{tabular}{ccccccc}
\hline methods & $\left|x_{2}-\alpha\right|$ & $\left|x_{3}-\alpha\right|$ & $\left|x_{4}-\alpha\right|$ & $\left|x_{5}-\alpha\right|$ & $f\left(x_{5}\right)$ & $C O C$ \\
$(1)$ & $0.114 \mathrm{e}-1$ & $0.162 \mathrm{e}-2$ & $0.363 \mathrm{e}-4$ & $0.117 \mathrm{e}-6$ & $0.117 \mathrm{e}-6$ & 1.6624 \\
$(2)$ & $0.106 \mathrm{e}-1$ & $0.217 \mathrm{e}-3$ & $0.937 \mathrm{e}-7$ & $0.176 \mathrm{e}-13$ & $0.176 \mathrm{e}-13$ & 2.0000 \\
$(62)$ & $0.245 \mathrm{e}-2$ & $0.856 \mathrm{e}-7$ & $0.893 \mathrm{e}-17$ & $0.341 \mathrm{e}-41$ & $0.341 \mathrm{e}-41$ & 2.4013 \\
$(7)$ & $0.246 \mathrm{e}-2$ & $0.696 \mathrm{e}-7$ & $0.990 \mathrm{e}-18$ & $0.168 \mathrm{e}-45$ & $0.168 \mathrm{e}-45$ & 2.5544 \\
$(63)$ & $0.246 \mathrm{e}-2$ & $0.977 \mathrm{e}-6$ & $0.213 \mathrm{e}-15$ & $0.157 \mathrm{e}-41$ & $0.157 \mathrm{e}-41$ & 2.7394 \\
$(64)$ & $0.246 \mathrm{e}-2$ & $0.924 \mathrm{e}-7$ & $0.198 \mathrm{e}-19$ & $0.167 \mathrm{e}-53$ & $0.167 \mathrm{e}-53$ & 2.7437 \\
$(8)$ & $0.883 \mathrm{e}-4$ & $0.689 \mathrm{e}-11$ & $0.407 \mathrm{e}-35$ & $0.131 \mathrm{e}-99$ & $0.131 \mathrm{e}-99$ & 2.8894 \\
$(9)$ & $0.247 \mathrm{e}-2$ & $0.109 \mathrm{e}-6$ & $0.966 \mathrm{e}-20$ & $0.140 \mathrm{e}-57$ & $0.140 \mathrm{e}-57$ & 2.8053 \\
$(10)$ & $0.899 \mathrm{e}-4$ & $0.715 \mathrm{e}-11$ & $0.586 \mathrm{e}-35$ & $0.786 \mathrm{e}-98$ & $0.786 \mathrm{e}-98$ & 2.8782 \\
\hline
\end{tabular}

\section{Conclusion}

In this work, we have developed four new three-point Secant-type methods for solving nonlinear equations with a simple root. The effectiveness of the new iterative methods is examined by showing the accuracy of the simple root of several nonlinear equations. Convergence analysis proves that the new three-point iterative methods preserve their order of convergence. Numerical test examples are provided to support the theoretical results obtained and compared with different methods. The major advantages of the new three-point Secant-type methods are that they are very effective, the new Secanttype iterative methods are shown to have a better order of convergence and efficiency index then the similar iterative methods considered. 


\section{REFERENCES}

[1] L. Chen, Y. Ma, A new modified King-Werner method solving nonlinear equations, Comp. Math. Appl. 62 (2011), 3700-3705

[2] G. Fernandez-Torres, A novel geometric modification of the Newton-secant method to achieve convergence of order $1+\sqrt{2}$ and its dynamics, Mod. Sim. Eng. 2015 (2015), art. ID 502854.

[3] D. E. Muller, A method for solving algebraic equations using an automatic computer. Math. Tab. Comput. 10 (1956), 208-215.

[4] A. M. Ostrowski, Solutions of equations and system of equations, Academic Press, New York, 1960.

[5] M. S. Petkovic, B. Neta, L. D. Petkovic, J. Dzunic, Multipoint methods for solving nonlinear equations, Elsevier 2012.

[6] H. Ren, A second-derivative-free modified Secant-like method with order 2.732... for unconstrained optimization, Appl. Math. Comput. 202 (2008), 688-692

[7] R. Thukral, A new secant-type method for solving nonlinear equations, Amer. J. Comput. Appl. Math. 8 (2) (2018), 32-36

[8] R. Thukral, Further development of secant-type methods for solving nonlinear equations, Inter. J. Adv. J. Math. 38 (5) (2018), 45-53.

[9] R. Thukral, New three-point secant-type methods for solving nonlinear equations, Amer. J. Comput. Appl. Math. 10 (1) (2020), 15-20.

[10] A.T.Tiruneh, W. N. Ndlela, S. J. Nkambule, A two-point Newton method suitable for nonconvergent cases and with super-quadratic convergence, Adv. Numer. Anal. 2013 (2013)Art. ID 687382.

[11] A.T.Tiruneh, T. Y. Debessai, G. C. Bwembya, S. J. Nkambule, A modified three-point secant method with improved rate and characteristics of convergence, Inter. J. Adv. Math. (4) (2019), 6983.

[12] J. F. Traub, Iterative Methods for solution of equations, Chelsea publishing company, New York 1977.

[13] X. Wang, J. Kou, C. Gu, A new modified secant-like method for solving nonlinear equations, Comp. Math. Appl. 60 (2010), 1633-1638

[14] X. Wang, J. Kou, C. Gu, A new modified secant-like method for solving nonlinear equations, Comp. Math. Appl. 60 (2010), 1633-1638 
[15] S. Weerakoon, T. G. I. Fernando, A variant of Newton's method with accelerated third-order convergence, Appl. Math. Lett. 13 (2000), 87-93. 